\title{
Modelling, Performance Analysis and Design of WPAN Systems
}

\author{
ANA GARCÍA-ARMADA, BEATRIZ BARDÓN RODRÍGUEZ, VÍCTOR P. GIL \\ JIMÉNEZ and MATILDE SÁNCHEZ-FERNÁNDEZ
}

University Carlos III de Madrid, Av. Universidad 30, 28911 Leganés, Spain

E-mails: \{agarcia, beatriz,vgil,mati\}@tsc.uc3m.es

\begin{abstract}
This paper covers the main issues that must be solved in order to design and analyse the performance of Wireless Personal Area Networks (WPANs) with the aid of simulation tools. We review state-of-the-art channel models to account for small- and large-scale propagation conditions in waveform- and system-level simulations. When dealing with waveform simulations, we also look at Montecarlo and importance sampling techniques that allow efficien estimation of error probabilities. Additionally, discrete channel models are introduced to efficientl link both waveform- and system-level approaches and we point out some methods to obtain the model parameters that are suitable for the wireless environment. The discussion of these techniques is complemented with two application examples that show the use of the different simulation levels for system design and performance study. With the paradigm of Bluetooth piconets we illustrate the usefulness of discrete channel models and we consider the design of an OFDM-based WPAN system to exemplify simulation from a waveform-level point of view.
\end{abstract}

Keywords: WPAN systems, simulation, discrete, channel, models, Bluetooth, H-OFDM

\section{Introduction}

The design of Wireless Personal Area Networks (WPANs) is challenging since these systems aim at achieving the best possible quality of service (QoS) while maintaining low-cost, low-power transceivers and making the most efficien use of the available bandwidth.

In this paper the main issues that must be solved in order to design and analyse the performance of WPANs with the aid of simulation tools are identifie and explained. Different performance parameters (Bit Error Rate (BER), frame error rate, throughput, delay) lead to different approaches from the simulation point of view, keeping always in mind the fl xibility and the efficien y required from these tools.

Waveform-level and system-level simulation are addressed and the ways to link both types of simulation are introduced. When approaching waveform-level simulation, short-term propagation channel models available for the suitable frequency bands are further reviewed in Section 2. Also, some techniques for estimation of BER are discussed in order to analyse the performance of the most frequent modulation techniques for these applications.

In Section 3, long-term propagation channel models are examined for system-level simulation. In order to link waveform- and system-level simulations, the use of discrete channel models with Hidden Markov Models (HMMs) is explained in Section 4. Discrete channel models with HMMs will efficientl represent the behaviour of the waveform-level, for different signal to noise ratios (SNR). This will be the key parameter to link both levels. System-level simulations will obtain the SNR through channel large scale effects and parameterise the 
HMM accordingly. Finally, two examples are provided as an application of these ideas and some conclusions are outlined.

Communication systems are becoming more complex every day. This makes their analytical treatment increasingly complicated in order to obtain the optimum system parameters, which, in general, require some performance parameter evaluation in non-ideal conditions.

On the other hand, the power of computers allows us to represent with increasing fidel ity the conditions in which real systems will be deployed using mathematical functions that constitute abstractions of the behavior of the systems under study.

People are surrounded or carry devices with a short-range coverage, usually limited to $10 \mathrm{~m}$. The WPAN systems are targeting ubiquitous communication of this kind. In order to build WPANs three main goals must be achieved: scalability, low-cost and low-power [1]. These communication systems should operate in very diverse environments in which the common characteristic is multipath propagation.

Simulation models will not only be used to evaluate the trade-offs between system design parameters but will also allow us to obtain an estimation of the performance of these systems prior to deployment and to evaluate the impact of inclusion of new technologies.

Simulation of communication systems is usually accomplished in a hierarchical fashion where several levels of abstraction can be define [2]:

Waveform-level simulation: the system is decomposed into several functional blocks and samples are generated representing the evolution of every signal from input to output.

System-level simulation: system topology in terms of nodes, links, queues, etc. is represented and events are being tracked through the system in order to estimate throughput and QoS parameters.

Figure 1 shows these different levels of hierarchical definitio of simulations.

An efficien interface between both simulation levels should provide a low-computational cost and time consuming representation of the Waveform-level. Discrete channel models based on HMMs will be used for this purpose providing an open interface through readily system-level parameters such as SNR and accurately matching the behavior of the lower level.

In the following sections some issues relevant to the simulation of WPAN systems in both levels of simulation are reviewed and Discrete Channel models are introduced in order to link waveform- and system-level simulations.

\section{Waveform-Level Simulation of WPAN Systems}

When setting up a waveform-level simulator we must represent the signals (waveforms) that are employed to transmit information from source to destination including modulation, filtering

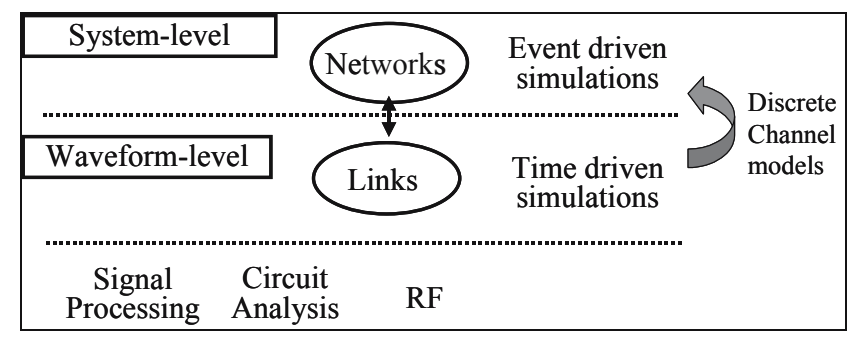

Figure 1. Hierarchical levels of simulation. 
amplification effects of the propagation channel, and interference. The accurate representation of each of these elements and the correct estimation of the signal parameters and error probabilities are of the utmost importance in order to obtain reliable results and this usually requires long simulations with several (i.e. 8-16) samples per symbol. In this paper, we will focus on two of the above mentioned important issues that must be considered when simulating Wireless Personal Area Networks at waveform-level: representing the influenc of the propagation channel in the signal and estimating the performance in terms of BER or Symbol Error Probability (SER). Different modulations are being used by different WPAN specifications therefore they will be explicitly considered in the examples given.

\subsection{Channel Models for Waveform-level Simulation of WPan}

The influenc of the propagation channel in wireless systems can be described in terms of two differentiated phenomena: large-scale and small-scale effects [3]. Depending of the hierarchical level of simulation, one of these types of effects must be considered. In this section, we deal with small-scale and in Section 3 large-scale effects will be examined.

Small-scale effects are due to multipath propagation that causes rapid variations of the received signal because of the addition of contributions arriving from different directions that have suffered different attenuations, delays, and Doppler shifts.

Small-scale effects are characterized by some parameters that must be known in order to adequately represent these effects in simulation:

- Coherence time.

- Maximum or RMS delay spread.

- Maximum Doppler shift.

- Coherence bandwidth.

These parameters, described in [3], are tightly related to the two phenomena that take place when the signal travels through a wireless channel: temporal or delay dispersion and Doppler dispersion. Figure 2

Modeling of these phenomena is accomplished through transversal filter with time-varying coefficients so that every filte stage represents the contribution of one of the multipath components.

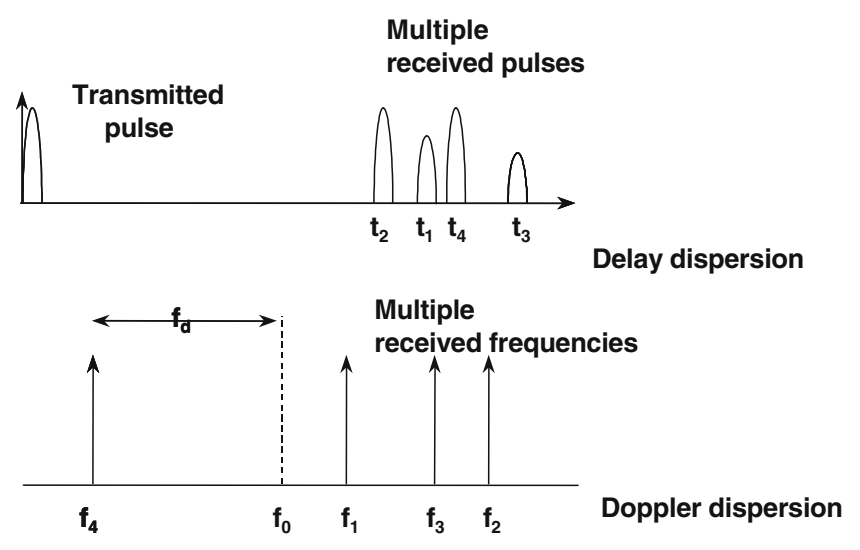

Figure 2. Small-scale effects. 
The variations in the received power from every path are represented by the channel powerdelay profil and are translated into the model by different weights in each tap of the tappeddelay line.

Time variations, characterized by the channel Doppler spectrum, are modeled through the variation of the filte coefficient along the simulation. With this goal in mind, filte coefficient are generated from complex random variables (reflectin amplitude and phase behavior) with the probability density function (pdf) according to the channel characteristics (Gaussian if channel envelope is Rayleigh) that are filtere in order to configur the Doppler spectrum of each contribution.

The selection of the number of coefficient is determined by the sampling frequency of the simulation and the maximum delay of the channel. As usual, a trade-off must be established between precision and computational cost.

Therefore, the parameters needed to implement the low-scale effects are: number of paths to be considered, amplitude, delay, phase, and Doppler characteristics of each of them.

These parameters depend on the application environment and the frequency band that is going to be used. For WPAN systems Industrial, Scientific and Medical (ISM) band of $2.4 \mathrm{GHz}$ is a good choice because of the following reasons [4]:

- License free: the ISM band in the $2.4 \mathrm{GHz}$ does not require the payment of any kind of taxes or fees for its public utilization.

- Propagation: the selection of higher frequencies is not strictly necessary for WPAN data rates, whereas it would have lead to higher propagation losses, increasing the required radiated power and thus the consumption and the cost of the power amplifie stage.

- Components cost: Due to the widespread use of this frequency band, the major manufacturers offer currently a wide range of components for the RF stages at a very low cost with more than reasonable features.

Propagation characteristics in this band are reported in [5] for several scenarios. No Doppler shift is considered in these scenarios since the mobility is highly restricted. As an illustrative example, let us consider two of these scenarios: a conference room (CR) and a laboratory room (LR). The dimensions of these environments are given in Table 1 (from [5]).

Figures 3-6 show the frequency response of two realizations of these channels obtained using a sampling frequency of $10 \mathrm{MHz}$. An exponential power delay profil is assumed and the number of taps used to model the channel is, together with the sampling frequency, dependent on the maximum delay spread of the channel.

Delay spread characteristics for these environments in Line-of-Sight (LOS) and Obstructed Line-of-Sight (OBS) conditions are shown in Table 2. These values are typical for indoor environments and have been taken from [5-7].

Table 1. Dimensions of the two example rooms

\begin{tabular}{lccl}
\hline Scenario & Length $(\mathrm{m})$ & Width $(\mathrm{m})$ & Height $(\mathrm{m})$ \\
\hline LR & 5.4 & 5 & 3.5 \\
CR & 27 & 10 & 3.5 \\
\hline
\end{tabular}


Table 2. Characteristics of the two example scenarios

\begin{tabular}{lcl}
\hline Scenario & RMS delay spread (ns) & Maximum delay spread (ns) \\
\hline LR LOS & 7.3 & 200 \\
LR OBS & 17.4 & 200 \\
CR LOS & 14.9 & 200 \\
CR OBS & 21.1 & 300 \\
\hline
\end{tabular}

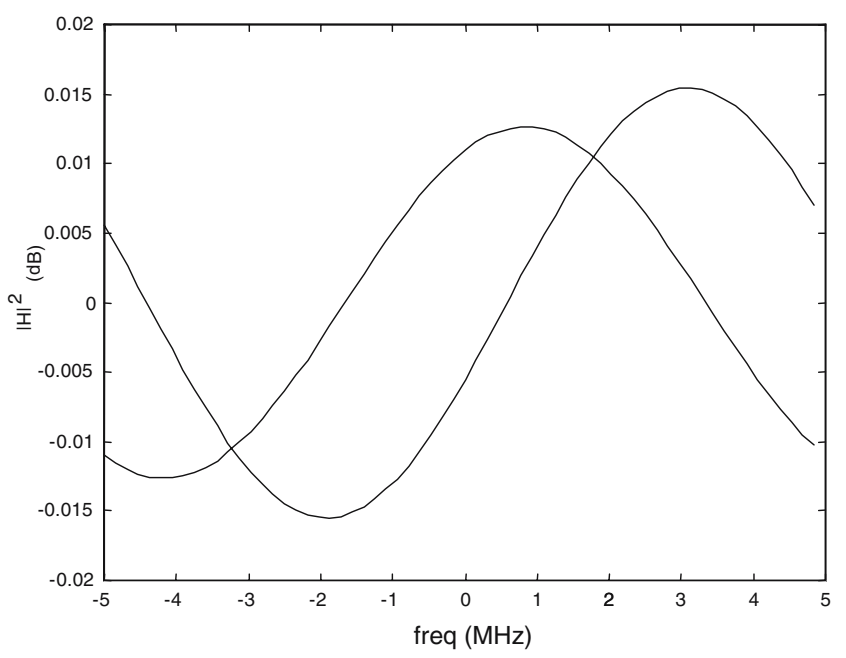

Figure 3. LR LOS with transmitting antenna at $3 \mathrm{~m}$ height.

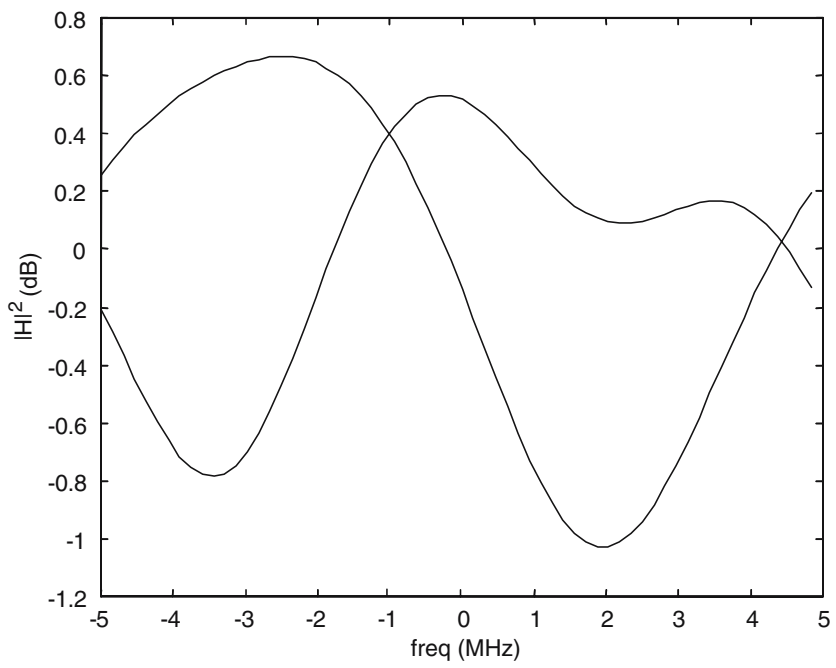

Figure 4. LR OBS with transmitting antenna at $1.5 \mathrm{~m}$ height.

\subsection{Monte Carlo estimation of Bit Error Probability}

Estimation of the error probability in communication systems via simulation is generally carried out using the well-known Monte Carlo method. However, some variance-reducing 


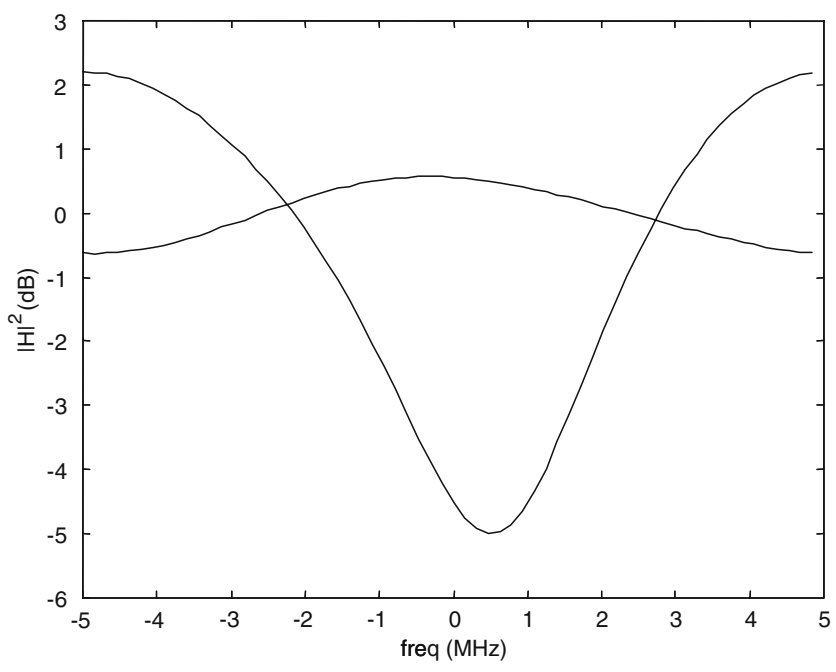

Figure 5. CR LOS with transmitting antenna at $3 \mathrm{~m}$ height.

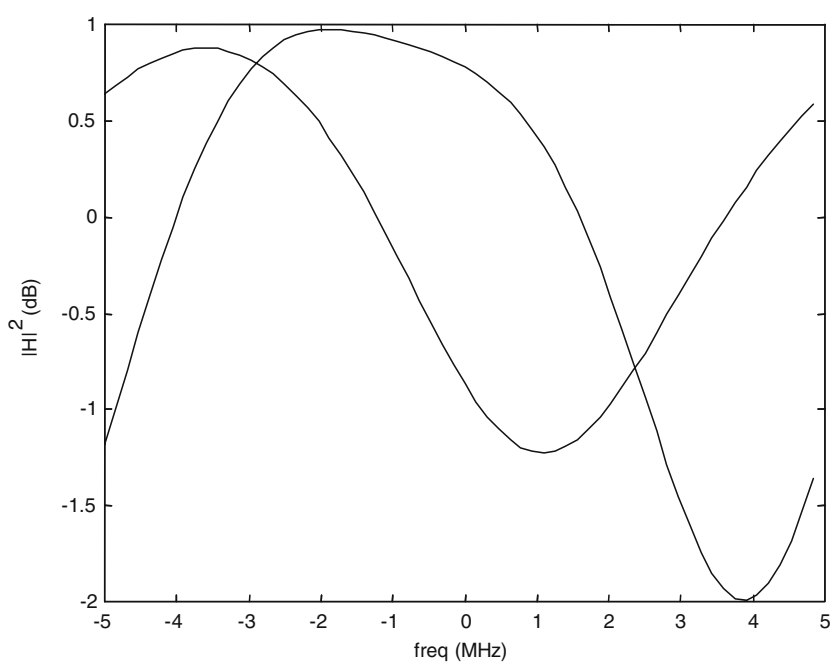

Figure 6. CR OBS with transmitting antenna at $3 \mathrm{~m}$ height.

techniques may be necessary in order to evaluate low-error probabilities while keeping the simulation time reduced to reasonable values.

The BER estimation using Monte Carlo has the advantage of applicability to any kind of communication system while some other techniques, such as semi-analytical BER estimation, require restrictive assumptions on the system. For this reason, it is the most commonly used technique.

We will illustrate the method considering the communication system whose block diagram is shown in Figure 7.

Let us assume that a given symbol is transmitted through the system and arrives to the receiver input. This is modeled with the random variable $X$ (this symbol can be either a binary information symbol: BER, or an M-ary information symbol: SER). The input decision variable at the decision device is $V$. 


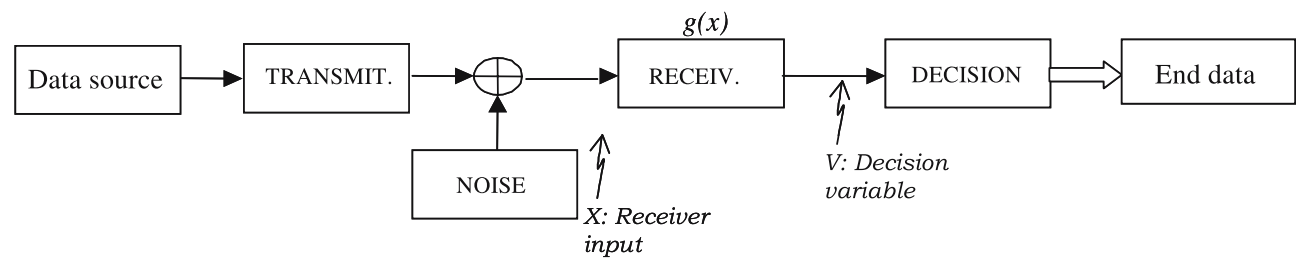

Figure 7. Block diagram of a communication system.

If we denote by $f_{V}(v)$ the pdf of the values that are found at the input of the decision device and $D_{\text {error }}$ the set of values of $V$ that will cause an erroneous decision, the error probability is given by:

$$
p=\int_{v \in D_{\text {error }}} f_{V}(v) d v
$$

If we defin an error indicating function $H(v)$ as:

$$
H(v)= \begin{cases}1, & v \in D_{\text {error }} \\ 0, & v \notin D_{\text {error }}\end{cases}
$$

then:

$$
p=E\{H(V)\}
$$

Therefore, the goal of estimation of $p$, that we will denote $\hat{p}$, is now formulated as obtaining an estimate of $H(V)$. The sample mean provides this estimation:

$$
\hat{p}=\frac{1}{N} \cdot \sum_{i} H\left(v_{i}\right)
$$

where:

- $N$ is the number of transmitted symbols.

- $H\left(v_{i}\right)$ indicates whether the decision is erroneous or not.

This estimator can be written in a simpler way if we denote by $n$ the number of observed errors that is, obviously, a function of the number of transmitted symbols $N$ :

$$
\hat{p}=\frac{n(N)}{N}
$$

It is important to consider the quality of this estimator. It can be shown [2] that this estimator is unbiased. Its variance will depend on the system memory.

If errors are generated independently, its variance can be found as [2]:

$$
\sigma^{2}(\hat{p})=\frac{p(1-p)}{N}
$$

However, if the system memory (define as the number of previous symbols that influenc a given output) is $m$, then the variance is:

$$
\sigma^{2}(\hat{p})=\frac{p(1-p)}{N} \cdot\left[1+2 m-\frac{m(m-1)}{N}\right]
$$


that can be simplified assuming $N \gg m$ as:

$$
\sigma^{2}(\hat{p}) \cong \frac{1}{N} \cdot p(1-p)(1+2 m)
$$

Table 3 shows some numerical examples of the number of symbols that must be simulated through the system in order to ensure a relative error in the estimation of $10 \%$ with different values of memory $m$. A multipath channel is a system with memory (given in our model by the number of sampled taps of the power-delay profile) From the values in Table 3, we can conclude that in cases of very low error probability and/or high memory some alternative methods for error estimation have to be considered in order to lower the simulation time.

Figure 8 shows the confidenc intervals of Monte Carlo estimator of the error probability [2]. In the ordinates error probability is written as a function of the parameter $v$ as:

$$
\hat{p}=10^{-v}
$$

\subsection{Importance Sampling estimation of Bit Error Probability}

It is clear now that some variance-reducing techniques may be necessary in order to evaluate low error probabilities of systems with memory while keeping the simulation time reduced to reasonable values. Importance Sampling is a modificatio of the classical Monte Carlo method that achieves this goal [8].

Table 3. Simulation length examples

\begin{tabular}{lll}
\hline$p$ & $N(m=0)$ & $N(m=100)$ \\
\hline $1,00 \mathrm{E}-02$ & 10,000 & 2010000 \\
$1,00 \mathrm{E}-04$ & $1,000,000$ & 201000000 \\
$1,00 \mathrm{E}-06$ & $100,000,000$ & $2,01 \mathrm{E}+10$ \\
$1,00 \mathrm{E}-08$ & $1 \mathrm{E}+10$ & $2,01 \mathrm{E}+12$ \\
\hline
\end{tabular}

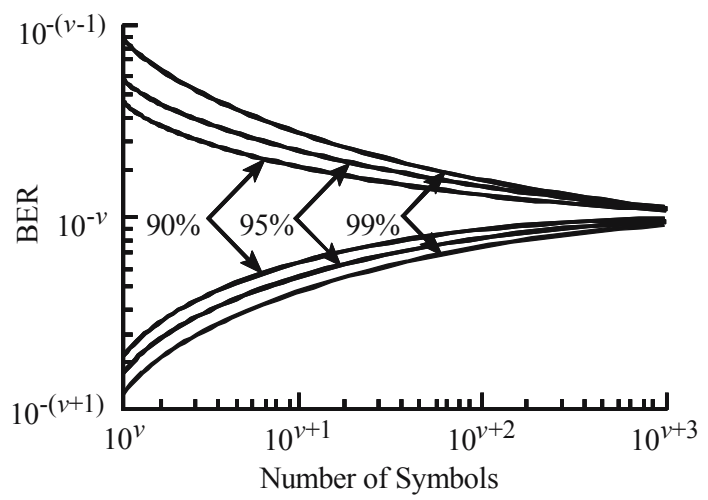

Figure 8. Confidenc intervals of MC estimator. 
The main idea is the introduction of some controlled changes in the signals so that the errors become more frequent and the variance of the estimator is decreased for the same simulation length.

Recalling the expression (3) of the error probability from the previous section:

$$
p=\int_{-\infty}^{\infty} H(V) \cdot f_{V}(v) \cdot d v
$$

it can be written in another way as a function of the input signals, being $g(x)$ the receiver impulse response and $f_{X}$ the pdf of $X$ (see Figure 7):

$$
p=\int_{-\infty}^{\infty} H(g(x)) \cdot f_{X}(x) \cdot d x
$$

If we now defin a weight function $w(x)$ as:

$$
w(x)=\frac{f_{X}(x)}{f_{X}^{*}(x)}
$$

Using this new function the error probability can be expressed as:

$$
p=\int_{-\infty}^{\infty} H(g(x)) \cdot w(x) \cdot f_{X}^{*}(x) \cdot d x
$$

where a new pdf $f^{*}$ is used in the calculations instead of the old one $f$. This is the essential idea of Importance Sampling: the input signals (noise and/or information) are modifie so that the pdf at the input of the receiver is changed in a known and controlled way.

If we call $E^{*}$ the expectation with respect to $f_{X}^{*}$, the former equation becomes:

$$
p=E^{*}\{H(g(X)) \cdot w(X)\}
$$

and again a natural estimation is the sample mean:

$$
\hat{p}_{I S}=\frac{1}{N} \cdot \sum_{i=1}^{N} H\left(g\left(\left(x_{i}\right)\right) \cdot w\left(x_{i}\right)\right.
$$

with $w\left(x_{i}\right)$ denoting the weighting function associated to the $i$ th symbol.

In a system with memory $m$ the weight for a given symbol $i$ must consider the preceding symbols as:

$$
w_{i}=\frac{f_{X}\left(x_{i}\right) \cdot f_{X}\left(x_{i-1}\right) \cdots \cdot f_{X}\left(x_{i-m+1}\right)}{f_{X}^{*}\left(x_{i}\right) \cdot f_{X}^{*}\left(x_{i-1}\right) \cdots \cdot f_{X}^{*}\left(x_{i-m+1}\right)}=\prod_{k=0}^{m-1} \frac{f_{X}\left(x_{i-k}\right)}{f_{X}^{*}\left(x_{i-k}\right)}
$$

Different Importance Sampling approaches can be found in the literature depending on how the input signals are modified

(a) Modificatio of the noise variance from $\sigma^{2}$ to $\sigma^{* 2}$, with $\sigma^{*}>\sigma$. This method is known as Conventional Importance Sampling (CIS). The problem is findin the optimum value of $\sigma^{*}$ that achieves the lowest variance of the estimator.

(b) Modificatio of the noise mean, known as Improved Importance Sampling (IIS).

(c) Simultaneous modificatio of the noise mean and variance. It is demonstrated in [9] that this simultaneous change of mean and variance does not imply any improvement and increases complexity. 
The improvement of CIS with respect to MC is given by the gain [2]:

$$
\eta=\frac{\left[N \cdot \sigma^{2}(\hat{p})\right]_{\mathrm{MC}}}{\left[N \cdot \sigma^{2}(\hat{p})\right]_{\mathrm{CIS}}}
$$

The modificatio of the noise variance is usually characterized by the following parameter:

$$
\gamma=\frac{\sigma^{*}}{\sigma}
$$

Figure 9 shows the values of the gain $\eta$ depending on the system memory and $\gamma$ when the system is linear, noise is AWGN and the target BER is $10^{-8}$. With the aid of Figure 9, optimum $\gamma$ can be obtained for a given simulation. More details can be found in [10].

\section{System-Level Simulation of WPAN Systems}

System-level simulations must be performed in order to obtain estimates of QoS parameters, such as throughput or delay, in a WPAN system consisting of several devices. In this section, we deal with large-scale channel models that can be used for this simulation approach. Those large scale models provide the parameters needed to be fed to lower levels of the simulator. The key parameter at this level is SNR or Signal-to-Interference-plus-Noise Ratio (SINR) that will have different variations for each device.

\subsection{CHANNEL MODELS FOR SYSTEM-LEVEL SIMULATION}

Large-scale propagation effects must be considered when performing system-level simulations in which simulation times are long enough so that slow variations of the signal are perceptible. They have a big influenc in coverage planning, decisions about access point emplacements [11] and design of power control algorithms.

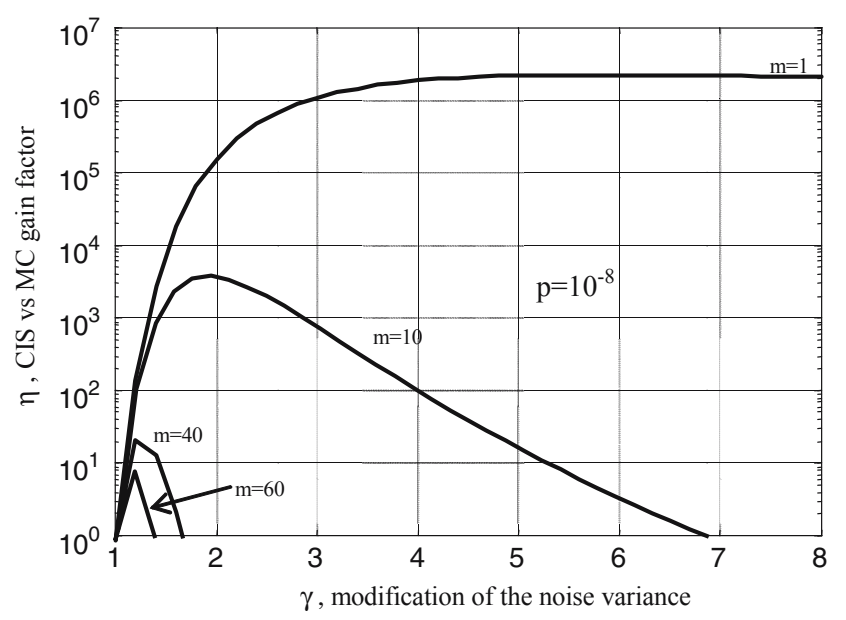

Figure 9. CIS improvement relative to MC. 
Large-scale effects are noticed as variations in the received power around a mean component that decreases as the distance between transmitter and receiver increases following the so-called path loss.

There exist numerous models in the literature that account for the path loss in different environments; well-known models are log-normal, Oukumura-Hata and some specifi ones developed within COST actions.

For WPAN systems in $2.4 \mathrm{GHz}$ ISM band some path loss expressions are provided in [5] that have been found by extensive measurements and respond to the following expression:

$$
P L(d B)=S_{0}+10 \cdot a \cdot \log _{10}(d)+b
$$

where $S_{0}$ is the path loss at $1 \mathrm{~m}$ distance, $a$ is the path loss exponent, $d$ the distance between transmitter and receiver in meters and $b$ is the value of the regression line at $d=1 \mathrm{~m}$. The measured value of $S_{0}$ is $43.1 \mathrm{~dB}$ at $2.4 \mathrm{GHz}$. Table 4 shows the values for the model parameters and the RMS error of the measured values with respect to the regression line for LOS and OBS situations.

For LOS situations the path loss exponent value is very close to the expected value for free-space. The value for $b$ is close to $0 \mathrm{~dB}$ and the RMS error is small.

For OBS situations the path loss exponent value increases. The calculated value for $b$ becomes increasingly negative and the RMS error also increases.

\section{Discrete Channel Models with HMMs}

Waveform-level simulations produce estimations of the BER or SER in some given environments. System-level simulations must make use of these values in order to characterize the behavior of the particular physical layer implementation of the devices that are sharing the network. However, it is desirable that as much information as possible coming from waveform-level simulations is fed back to system-level simulations. In this section, we explore how waveform- and system-level simulations may be linked through the use of Discrete Channel models.

Finite state channel models [3] are a useful tool for simulating communication systems operating over fading channels. The bursty nature of errors generated in that type of channels is reproduced by means of a state diagram that avoids the simulation of the whole physical channel. Moreover, when high quality transmission is required the inclusion of some kind of channel coding can be necessary. The BER involved in that case will imply the use of an efficien simulation model to optimise the simulation computational cost.

Figure 10 shows a block diagram of a general communication system operating over a wireless channel. The lowest level (level 1) represents the part of the system associated with the waveform signal, that is, the set composed of modulators, filters equalizers and the radio

Table 4. Model parameters for $2.4 \mathrm{GHz}$ ISM band

\begin{tabular}{llll}
\hline Scenario & $a$ & $b(\mathrm{~dB})$ & RMS error $(\mathrm{dB})$ \\
\hline LOS & 1,86 & -1.6 & 1.6 \\
OBS & 3.33 & -5.4 & 3.6 \\
\hline
\end{tabular}




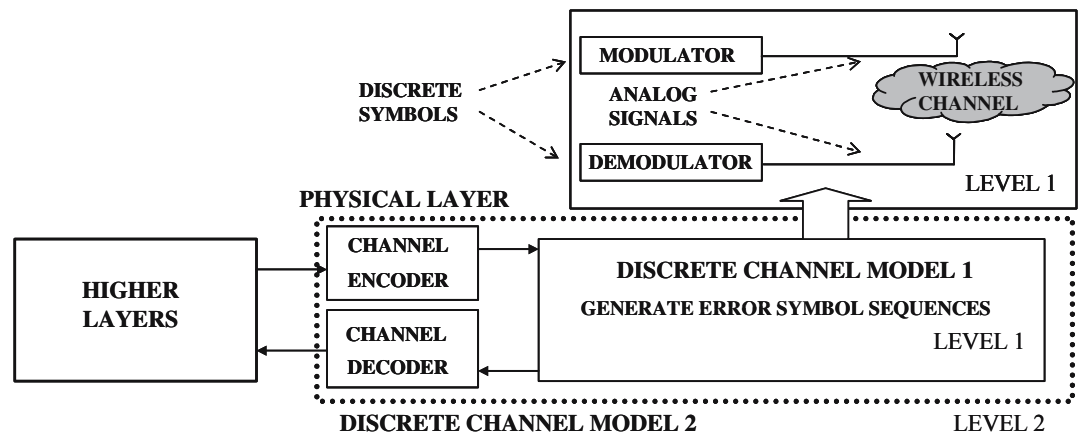

Figure 10. Nested levels for efficien simulation.

channel. Due to effects such as fading and multipath the generated errors will have a bursty nature.

Taking into account that for the next level the firs one can be seen as a bit error generator it would be desirable to obtain an efficien simulation model by simply replacing the lowest level by a black box that is able to generate errors with the same statistical characteristics as the ones originated from the real system.

Subsequently, in order to optimize the simulation of the complete physical layer, a second level including channel coding can be established. If we are able to obtain a discrete channel model nested with the previous one we would have a very useful tool to test, once the simulation models have been validated, the different applications using the physical layer.

The parameters that describe a HMM are grouped into four sets [2]:

- Set of $N$ states, each one of them modelling the different possibilities of behaviour of the channel.

- Set of state probabilities $\pi_{\mathrm{i}}(t)$ that characterise the probability that the channel is in state $i$ at time $t$ :

$$
\pi_{\mathrm{i}}(t) P\left[S_{t}=i\right], \quad i=1,2, \ldots, N
$$

- Set of state transition probabilities $a_{i j}(t)$ describing the probability of the channel changing from one state to another:

$$
a_{i j}(t)=P\left[S_{t+1}=j \mid S_{t}=i\right]
$$

- Set of symbol error probabilities $b_{i}\left(e_{k}\right)=b_{i k}$ for every state:

$b_{i}\left(e_{k}\right)=P\left[e_{k} \mid S_{t}=i\right]$, with $e_{k}$ denoting one of the $M$ possible error symbols $E=$ $\left\{e_{1}, e_{2}, \ldots, e_{M}\right\}$.

The dependence of the HMM parameters with time $t$ represents the variability that they will have based on the external parameters provided by the system level simulator.

The output of the HMM will consist of two sequences, the sequence of successive channel states $S_{i}$ and the sequence of error symbols $e_{k}$ generated by the model.

In [2] it is shown that under certain conditions all the parameters can be inferred from the estimation of two matrices: the state transition matrix $\mathbf{A}$ and the input to output transition probabilities matrix $\mathbf{B}$ (the dependence on both matrices with $t$ is dropped for the sake of 
simplicity) of the form:

$$
\mathrm{A}=\left[\begin{array}{ccc}
a_{11} & \ldots & a_{1 N} \\
\cdot & & \cdot \\
\cdot & & \cdot \\
a_{N 1} & \ldots & a_{N N}
\end{array}\right] \quad \mathrm{B}=\left[\begin{array}{ccc}
b_{11} & \ldots & b_{1 M} \\
\cdot & & \cdot \\
\cdot & & \cdot \\
b_{N 1} & \ldots & b_{N M}
\end{array}\right]
$$

Figure 11 shows an example of a state diagram for an HMM with $N=3$.

Starting from a long enough error sequence obtained from the lowest level of simulation and using the iterative procedure known as the Baum-Welch algorithm [2] it is possible to obtain a pair of matrices that can be used to generate error sequences with the same characteristics as the one used to train the algorithm. Once we have obtained these matrices, the model to be used in upper level of simulation is characterised.

The direct application of the Baum-Welch algorithm can require a very great amount of computations, specially when the error sequence contains long chains of identical symbols. Moreover, every time simulation conditions change a new set of parameters must be obtained so the optimisation of the algorithm becomes more relevant. In [12] it is presented the way for obtaining a Markov model equivalent to the one represented by matrices $\mathbf{A}$ and $\mathbf{B}$. In this model the state transition probability matrix is converted into an equivalent "block diagonal" matrix $\boldsymbol{\Lambda}$, which is estimated using a modifie version of the Baum-Welch algorithm that involves great computational savings. This efficien approach is adequate for WPAN channels.

\section{Examples}

Two different examples show each of the parts of the design so far presented. The firs example deals with the construction of a discrete channel model for Bluetooth piconets and the second one with the performance study at waveform-level of an OFDM-based WPAN.

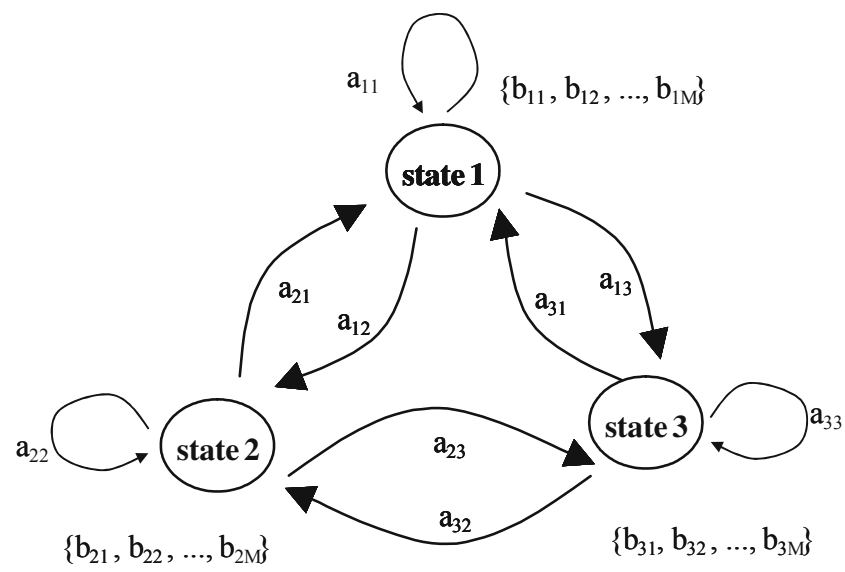

Figure 11. State diagram for a three states Markov model. 


\subsection{BLUETOOTH PICONETS}

Let us consider the development of Discrete Channel models for a particular WPAN scenario configure by Bluetooth devices that communicate within a given piconet (piconets are lower level networks that constitute the building blocks of scatternets in Bluetooth terminology) [13].

In order to obtain the model parameters some complete waveform-level simulations must be run. The output of each of these Monte Carlo simulations is an error burst that reflect the behaviour of the elements included in the link-level simulation. Following Bluetooth specification $[14,15]$, simulations include the following parameters:

- Gaussian BFSK modulation with bandwidth times bit-time product BT $=0.5$ and modulation index $0.28<h<0.35$

- 1/3, 2/3 FEC codes for synchronous connections; 2/3 FEC for asynchronous connections with multi-slot capability. Also an uncoded transmission and different interleaving options are investigated.

Figure 12 shows the system block diagram used to perform waveform-level simulations.

Using both Baum-Welch and the modifie algorithm of [12] and starting from the error sequence generated in the simulation of the Bluetooth physical layer, the parameters that characterize the two models have been obtained in [13]. Figure 13 shows the transition matrix obtained for SNR of $4 \mathrm{~dB}$. The matrix is divided in four parts: upper-left block diagonal represents the probability of staying at each of the "good" states; down-right diagonal represents the probability of staying at each of the "bad" states; upper-right and down-left represent the transition probabilities from "good" to "bad" states and conversely.

In order to validate the proposed models several simulations have been run obtaining the burst error sequences generated by the two HMMs. These sequences have been compared with the ones arising from the original physical layer. To make this comparison the two

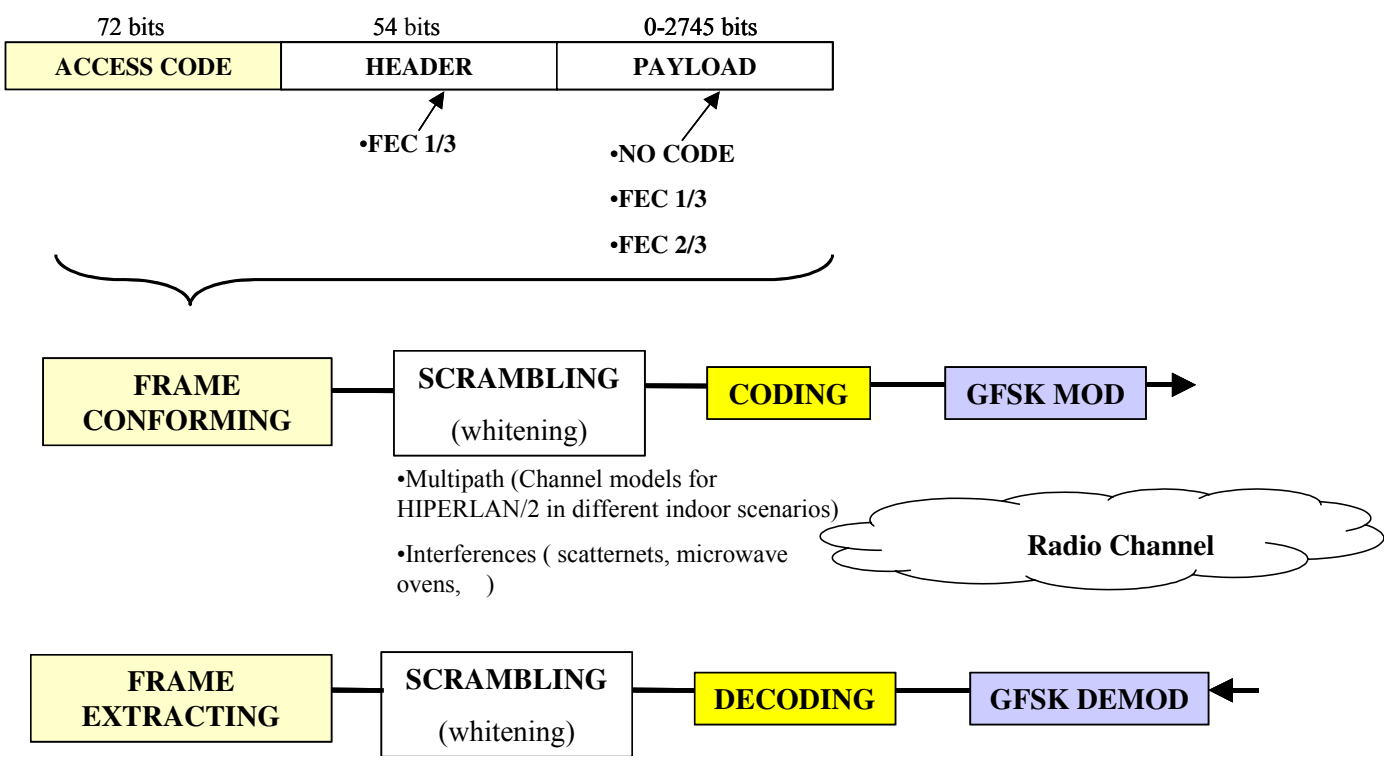

Figure 12. Bluetooth waveform-level simulation block diagram. 


\begin{tabular}{cccccc|cccccc}
0.7985 & 0 & 0 & 0 & 0 & 0 & 0.0015 & 0.0269 & 0.0017 & 0.0346 & 0.1346 & 0.0021 \\
0 & 0.7923 & 0 & 0 & 0 & 0 & 0.0433 & 0.0132 & 0.0007 & 0.1315 & 0.0075 & 0.0114 \\
0 & 0 & 0.7182 & 0 & 0 & 0 & 0.0464 & 0.0309 & 0.0000 & 0.1616 & 0.0282 & 0.0147 \\
0 & 0 & 0 & 0 & 0 & 0 & 0.0000 & 0.0000 & 1.0000 & 0.0000 & 0.0000 & 0.0000 \\
0 & 0 & 0 & 0 & 0.7975 & 0 & 0.0224 & 0.0031 & 0.0005 & 0.0675 & 0.0789 & 0.0302 \\
0 & 0 & 0 & 0 & 0 & 0.8376 & 0.0026 & 0.0426 & 0.0000 & 0.0715 & 0.0224 & 0.0233 \\
\hline 0.0024 & 0.0201 & 0.0220 & 0.0000 & 0.0560 & 0.6881 & 0.2115 & 0 & 0 & 0 & 0 & 0 \\
0.0124 & 0.0130 & 0.0115 & 0.0000 & 0.0362 & 0.7075 & 0 & 0.2193 & 0 & 0 & 0 & 0 \\
0.0006 & 0.0225 & 0.9495 & 0.0000 & 0.0273 & 0.0000 & 0 & 0 & 0.0000 & 0 & 0 & 0 \\
0.0049 & 0.0031 & 0.0030 & 0.0000 & 0.0480 & 0.7240 & 0 & 0 & 0 & 0.2169 & 0 & 0 \\
0.0048 & 0.0281 & 0.0062 & 0.0000 & 0.0554 & 0.6864 & 0 & 0 & 0 & 0 & 0.2190 & 0 \\
0.0166 & 0.0418 & 0.0094 & 0.0000 & 0.0897 & 0.6201 & 0 & 0 & 0 & 0 & 0 & 0.2225
\end{tabular}

Figure 13. Transition matrix for Bluetooth with $\mathrm{SNR}=4 \mathrm{~dB}$.

sequences are cross-correlated. Moreover, the histograms characterising the error free intervals for the different guard times are obtained and latter compared to the ones in the original error sequence. The goodness-of-fi test used for comparing the similarity of both histograms has been the chi-square [13].

Once validated, these methods can be used to generate error sequences that the decoder will process, allowing the analysis of trade-offs between the different coding and interleaving schemes with a reasonably consumption of computational resources.

Some other examples of the use of HMMs for the simulation of wireless systems, not specificall WPANs though, can be found in [16-18].

\subsection{OFDM-BASED WPAN}

Power Aware Communications for Wireless OptiMised personal Area Network (PACWOMAN) project has been working on solutions in order to build WPANs that achieve three main goals: scalability, low cost and low power [19]. With these in mind, a hybrid OFDM-based multiple access scheme has been proposed, H-OFDM, that is a combination of OFDMA and TDMA, i.e., the OFDM signal is frequency-shared and time-shared.

The design of the main parameters of the H-OFDM signal is described in [1]. Depending on the data rates, terminals are divided in three categories, namely, Low Data Rate (LDR) ranging from 0.01 to $10 \mathrm{kbps}$, MDR (Medium Data Rate) from 0.01 to $1 \mathrm{Mbps}$ and High Data Rate (HDR) for speeds of $1 \mathrm{Mbps}$ or above. H-OFDM is targeting MDR/HDR terminals and the main parameters chosen for the signal are shown in Table 5.

Waveform-level simulations have been performed for this H-OFDM system using the channel models described in section 3. As an example, Figure 14 shows the performance of two possible channel estimation schemes in a multi-user environment (MAE: channel estimation based on Multi-Antenna theory and FD: channel estimation based on Frequency-Division of the preamble).

Figure 15 shows the global performance of H-OFDM considering an 8-user environment when one user is perfectly synchronized with another and the rest have small misalignments: 1 sample-delay (users 3 and 8), phase noise of $10^{-3} \operatorname{rad}^{2}$ (users 2 to 4 ) and $10^{-4} \mathrm{rad}^{2}$ (users 5 and above) and small frequency offset (users 4 to 6 ). 
Table 5. Main parameters of H-OFDM

\begin{tabular}{ll}
\hline Parameter & Value \\
\hline Number of sub-carriers $(N)$ & 64 \\
Number and distribution frequency guards & 8 at each boundary \\
Number and distribution of pilots & Full-pilot preamble; four scattered in every symbol \\
Number of useful sub-carriers & 44 \\
Bandwidth & $10 \mathrm{MHz}$ \\
Duration of cyclic prefi & $400 \mathrm{nsec} .(4$ samples $)$ \\
Duration of useful OFDM symbol & $6.4 \mu \mathrm{sec}$ \\
Number of slots per frame $(L)$ & 16 \\
Slot length & $1.05 \mathrm{msec}\left(N_{O F D M}=150\right)$ \\
$R_{b}$ (data rate) & $9.2 \mathrm{Kbps}-38.8 \mathrm{Mbps}$ \\
\hline
\end{tabular}

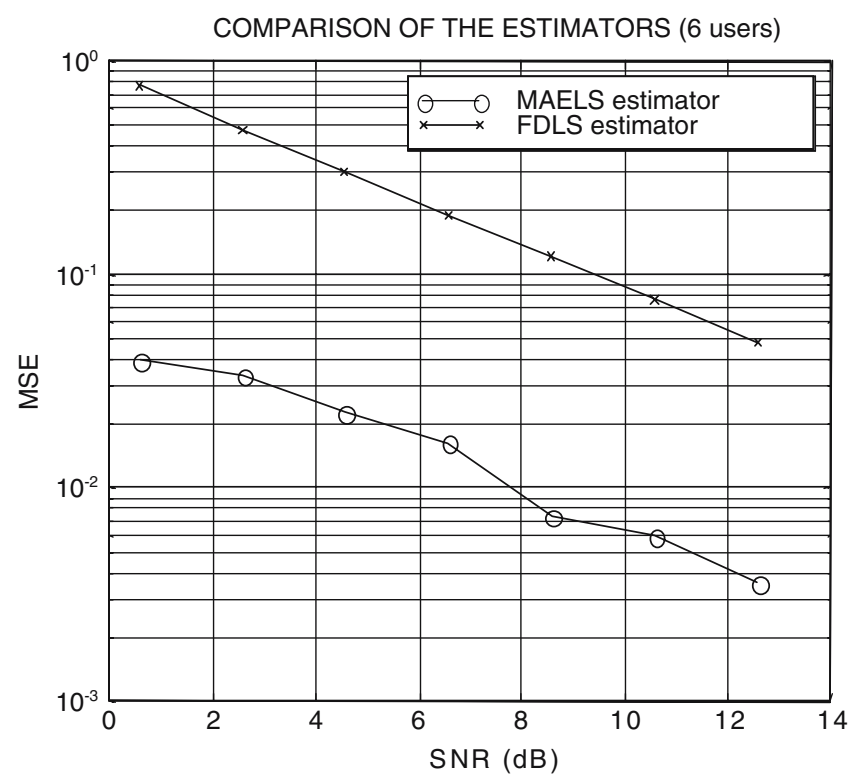

Figure 14. H-OFDM of channel estimators.

These simulations allow us to design the system, select the optimum parameters and choose the best algorithms to obtain the required performance in the receivers.

\section{Conclusions and Research Visions}

Simulation of communication systems is a mature area. Channel models and performance evaluation have been dealt with in the past and an extensive literature can be found on the topics.

In opinion of the authors, research must continue, however, in two specifi areas:

- Efficien simulation of very low error probabilities. With this goal in mind, solutions must be found in order to apply some Importance Sampling techniques to coded systems in which exhaustive simulation of the waveform is computationally prohibitive (such as Turbo-coded communication systems). 


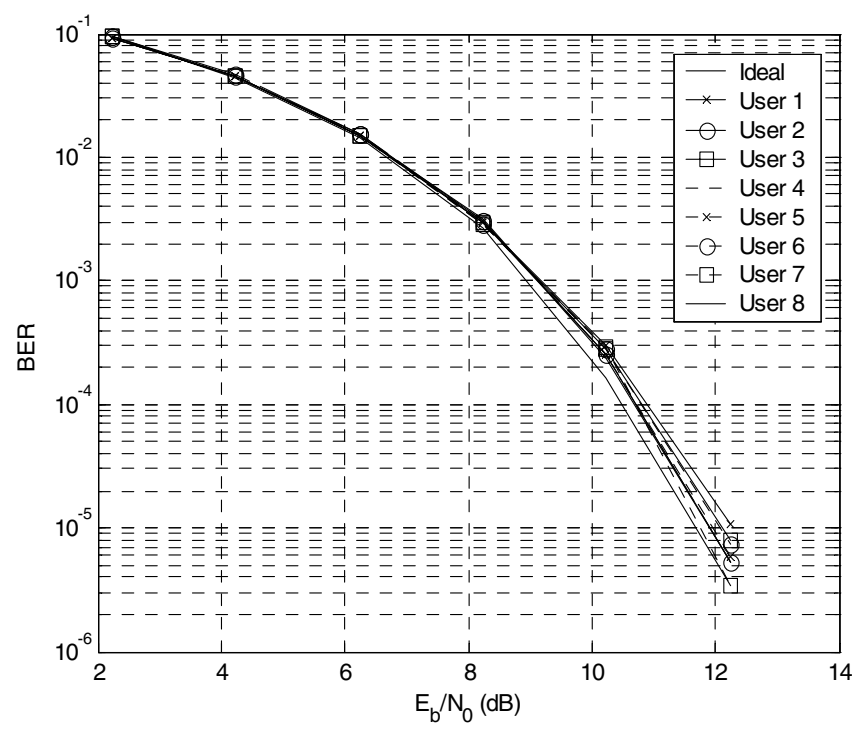

Figure 15. H-OFDM global performance.

- Obtaining discrete channel models via HMMs for wireless communications systems (and specificall for WPANs) in order to feed-back more information than just BER or SER from waveform-level to system-level will improve the efficien y of these systems in which constraints on cost, power and information rate are usually tight.

The design of WPANs is challenging since these systems aim at achieving the best possible QoS will maintaining low-cost, low-power transceivers, and making the most efficien use of the available bandwidth.

In this paper the main issues that must be solved in order to design and analyse the performance of WPANs with the aid of simulation tools have been identified explained and illustrated with two examples.

Simulations allow us to design WPAN systems, select the optimum parameters and choose the best algorithms to obtain the required performance in the receivers.

Two open issues remain for further research, namely, efficien simulation of very-low-error rate systems and obtaining Discrete Channel models for WPAN and, in general, wireless systems.

The efficien simulation of coded communication systems (such as Turbo-Codes) will not only be important for the better deployment of WPAN systems but to communication systems in general, both wired and wireless.

The same applies to the findin of advanced methods to estimate Discrete Channel models for communication systems. These models will make it possible to test higher layer protocols and applications under more realistic conditions, bringing more efficien y to them and allowing the test of trade-offs in what is referred as cross-layer design.

\section{Acknowledgements}

This work has been partially funded by European Commission (IST-2001-34157), Spanish Government (TIC2002-03498) and Madrid Community (UC3M-TEC-05-027). 


\section{References}

1. A. García Armada, V. P. Gil Jiménez, M. J. Fernández-Getino García and J. L. García. "H-OFDM design for Wireless Personal Area Communications”, Proceeding of IST Mobile \& Wireless Communications Summit, Aveiro, Portugal, Vol. I, pp. 93-97, 2003.

2. M. Jeruchim, P. Balaban and K. S. Shanmugan, Simulation of Communication Systems, 2nd edn. New York, Plenum Publishing Corp. 2000.

3. T. S. Rappaport, Wireless Communications. Principles \& Practice, Englewood Cliffs, NJ, Prentice Hall, 1996.

4. A. García Armada, M. J. Fernández-Getino, V. P. Gil Jiménez, M. Lobeira, J. C. González. J. L. García and B. Bougard. "Analysis and simulation of physical layer requirements. Synchronisation, PAR reduction and resource assignment algorithms. RF specifications" IST-2001-34157, Deliverable D.4.2.3, September 2003. www.imec.be/pacwoman.

5. G. J. M. Janssen, P. A. Stigter and R.Prasad, "Wideband Indoor Channel Measurements and BER Analysis of Frequency Selective Multipath Channels at 2.4, 4.75, and 11.5 GHz”, IEEE Transactions on Communications, Vol 44. No. 10, pp. 1272-1288, 1996.

6. A. A. Saleh and R. A. Valenzuela, "A Statistical Model for Indoor Multipath Propagation", IEEE Journal on Selected Areas in Communications, Vol. 5, No. 2, pp. 128-137, 1987.

7. W. M. Teck and L. C. Look, "Wideband impulse response of indoor channel at $2 \mathrm{GHz}$ using directive patch antennae", IEEE International Conference on Information Engineering (ICIE), pp. 532-536, Singapore, 1995.

8. M. C. Jeruchim, "Techniques for Estimating the Bit Error Rate in the Simulation of Digital Communication Systems", IEEE Journal on Selected Areas in Communications, Vol. 2, No. 1, pp. 153-170, 1984.

9. D. Lu and K. Yao, "Improved Importance Sampling Technique for Efficien Simulation of Digital Communication Systems”, IEEE Journal on Selected Areas in Communications, Vol. 6, No. 1, pp.67-74, 1988.

10. P. Hahn and M. C. Jeruchim, "Developments in the Theory and Application of Importance Sampling", IEEE Transactions on Communications, Vol. COM-35, No. 7, pp. 706-714, 1987.

11. A. J. Motley and J. M. P. Keenan, "Personal communication radio coverage in buildings at $900 \mathrm{MHz}$ and 1700 MHz.”, Electronic Letters, Vol. 24, No. 12, pp. 763-761, 1988.

12. S. Sivaprakasam and K. S. Shanmugan, "An equivalent Markov model for burst errors in digital channels", IEEE Transactions on Communications, Vol. 43, No. 2/3/4, pp. 1347-1353, 1995.

13. B. Bardón, M. Sánchez and A. García, "Discrete Channel Simulation of Bluetooth Piconets", International Workshop on Broadband Wireless Ad-Hoc Networks and Services, ETSI, Sophia Antipolis, Francia, September 2002.

14. S. Mattisson et al, "Bluetooth Core Specifiations" Bluetooth SIG, February 2001.

15. J. C. Haarten et al., "The Bluetooth Radio System”, IEEE Personal Communications, Vol. 7, No. 1, pp. 28-36 February 2000.

16. K.S. Shanmugan and A. Beverly, "Hidden Markov Models for Burst Errors in GSM and DECT Channels", Proceedings of Globecom '98, Vol. 6, pp. 3692-3698, Sydney, Australia Nov. 1998.

17. P. Kuczynsky et al., "Hidden Markov Modeling of Error Patterns and Soft Outputs for Simulation of Wideband CDMA Transmission Systems", European Conference on Wireless Technology (ECWT), pp. 135-138, Paris, October 2000.

18. A. Umbert et al., "A Radio Channel Emulator for WCDMA Based on the Hidden Markov Model (HMM)", IEEE Vehicular Technology Conference (VTC), pp. 2173-2179, Boston, September 2000.

19. F. Catthoor, L. Deneire, J. Ayadi, A. Hutter, C. Wijting, L. Gavrilovska, H. Yomo, E. Metin, J. Choque, M. García, V. Gutiérrez, J. Irastorza, L. Muñoz, R. Sanz, S. Kyriazakos, V. Owall and R. Velentzas, T. Charity, J. Spanoudakis, "System Requirements and Analysis", PACWOMAN (IST-2001-34157) Deliverable, D2.1, June 2002, www.imec.be/pacwoman. 


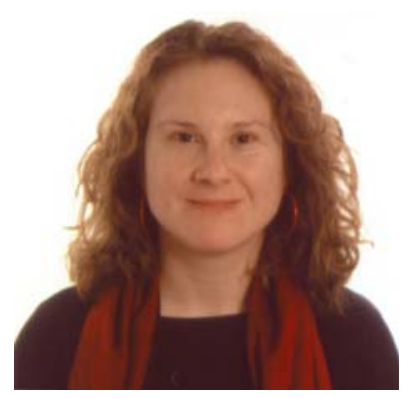

Ana García Armada received the Telecommunication Engineer degree and the Ph. D. in Electrical Engineering both from the Polytechnic University of Madrid (Spain) in 1994 and 1998 respectively. She is currently working as an Associate Professor at the University Carlos III de Madrid, where she has occupied several management positions. She has participated in several national and international research projects, most of them related to OFDM. She is co-author of four books on wireless communications and signal processing. She has published 15 papers in international journals and more than 40 papers in conferences. She has contributed to international organizations such as ITU and ETSI. She has performed research stays in ESA-ESTEC, Kansas University, Stanford University and Bell Labs. Her research interests are simulation of communication systems, multicarrier and MIMO techniques.

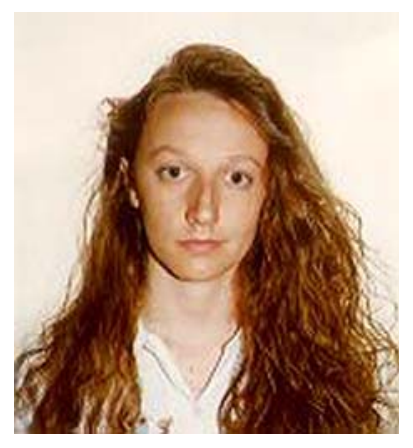

Beatriz Bardón Rodríguez received the M. Eng. degree in Telecommunications from the Politecnic University de Madrid in 1998. Actually she is working towards the Ph. D. She is with the Department of Signal Theory and Communications at the University Carlos III de Madrid as an Assistant Professor. Her research interests include Hidden Markov Models, simulation of wireless communication systems and signal processing for communications. 


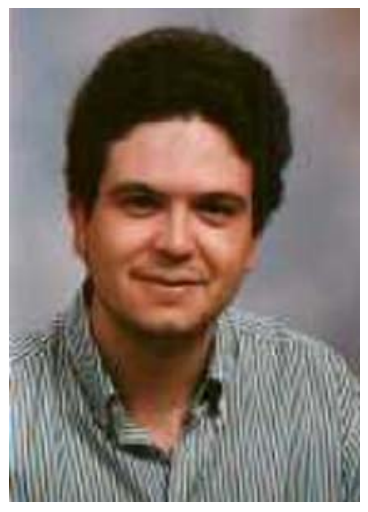

Víctor P. Gil Jiménez received the B. Eng. in Telecommunications with honors from University of Alcalá in 1998 and the M. Eng. in Telecommunications and the PhD. degree both from the University Carlos III de Madrid in 2001 and 2005 respectively. He is with the Department of Signal Theory and Communications at the University Carlos III de Madrid as an Assistant Professor. He worked at the Spanish Antarctica Base in 1999 as Communications Staff. He visited University of Leeds and Chalmers Technical University in 2003 and 2004 respectively. His research interests include multicarrier communications and signal processing for wireless systems.

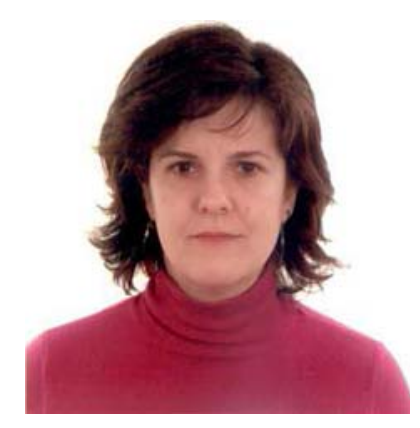

Matilde P. Sánchez Fernández obtained her Telecom. Engineer degree from Technical Univ. of Madrid in 1996, and her Doctor degree (Honors) from Technical Univ. of Madrid in 2001. She is an Assistant Professor at University Carlos III de Madrid, Spain since April 2000. Previously, she worked for Telefónica as a Telecommunication Engineer. She has performed several research stays at the Information and Telecommunication Technology Center in Kansas University and at Bell-Labs, New Jersey. Her current research interests are dealing with MIMO techniques, Turbo Codes, mobile communications, simulation and modelling of communication systems, and Adaptive Coding \& Modulation. 